\title{
Simulation of PLCC Routing Algorithm using OPNET Modeler
}

\author{
J.C. Chen, R.Q. Yan, Y.N. Zhang, Y.H. Zhao \\ College of Automation Engineering \\ Northeast Dianli University \\ China
}

\begin{abstract}
Today, the power line carrier communication (PLCC) technology has become a research focus in the field of communication. It is important to study on the PLCC routing algorithms, since the power line carrier channel has the characteristics of high time-variation, high noise and high attenuation. The PLCC routing algorithms that have been proposed, such as the artificial spider web routing algorithm, ant-colony based routing algorithm, and flooding algorithm etc., are feasible in principle, but their actual performance is not clear. In this paper, the method for simulation on a PLCC routing algorithm which is similar to the dynamic source routing algorithm, using OPNET, is discussed. Not only the methods for OPNET modelling but also the experimental results are presented in the paper. They have important reference values for design of a PLCC network.
\end{abstract}

Keywords-routing algorithm; PLCC; simulation; OPNET

\section{INTRODUCTION}

The power line carrier communication (PLCC) technology comes into being in the early 1920s. The PLCC systems use the existing home electrical wiring for the transformation of information. It becomes easy to develop a home automation system or connect the houses with a high speed network access point without installing new wirings [1]. With a decade of efforts, the PLCC technology is continuously developing, and the application of the PLCC technology also gradually becomes a research focus [2]. Because the PLCC technology has some competitive advantages, such as free maintenance, less investment, high safety and reliability, plug and play, synchronous with power grid construction etc., it has been widely used in industrial control system, home automation system, automatic meter reading system, etc. [3].

It is important to study on the PLCC routing algorithms, since the power line carrier channel has the characteristics of high time-variation, high noise and high attenuation[4]. The proposed PLCC routing algorithms, such as artificial spider web routing algorithm[5], ant colony based routing algorithm[6,7], and flooding algorithm[8], are feasible in principle, but their actual performance is not clear. In this paper, the method for simulation on a PLCC routing algorithm which is similar to the dynamic source (SDS) PLCC routing algorithm, using OPNET, is discussed. Not only the methods for OPNET modelling but also the experimental results are presented in the paper.

\section{PRINCIPLE OF THE SDS PLCC ROUTING ALGORITHM}

The SDS routing algorithm is only suitable for a masterslave PLCC network. This method is realized by saving a regular dynamic updated routing table in a master. Before sending packets, the master will first search the routing table. The slaves do not store the routing table, but receive or forward the packets according to the destination address field [9].

An example is used to explain the principle of the SDS PLCC routing algorithm. As shown in Fig. 1, a master-slave PLCC network is composed of one master (node 0 ) and nine slaves (node 1 to node 9).

To build a routing table, a $10 \times 10$ zero matrix (transmission matrix) $\mathrm{M}$ is first built, and then the master sends a broadcast packet. The slaves that receive the broadcast packet will send a response packet with their address information. Suppose that the broadcast packet is only received by the slaves 2,3 , and 5 , then the elements $\mathrm{M}(0,2), \mathrm{M}(0,3)$, and $\mathrm{M}(0,5)$ of the transmission matrix $\mathrm{M}$ are set to one, after analyzing the response packets by the master. On this basis, the master sends a command packet to the slave 2 , and the slave 2 send a broadcast packet after receiving the command packet. The slaves, which receive the broadcast packet sent by the slave 2 and have not sent a response packet to the master, will send a response packet to the slave 2 . If the slaves 1 , and 6 receive the broadcast packet sent by the slave 2 , they will send response packets to the slave 2 , and the slave 2 will send a response packet, which includes the address information of the slaves 1 and 6 , to the master. At last, the elements $\mathrm{M}(2,1)$ and $\mathrm{M}(2,6)$ of the transmission matrix $\mathrm{M}$ will be set to one by the master.

The previous procedure will continue until the address information of all the slaves is received by the master. The final created transmission matrix is shown in Fig. 2. On this basis, the network topology of the PLCC network is also created as shown in Fig.3.

After initialization is completed, the master can send a message packet to any slave according to the transmission matrix. If the master wants to send a message packet to the slave $\mathrm{j}$, it would search the jth column of the transmission matrix to find which element's value is equal to one. For example, if the value of the element $\mathrm{M}(\mathrm{i}, \mathrm{j})$ is equal to one, the slave $i$ is the repeater that receives a message packet and retransmits it to the slave $\mathrm{j}$. This procedure will continue until the master is found to be a repeater. 


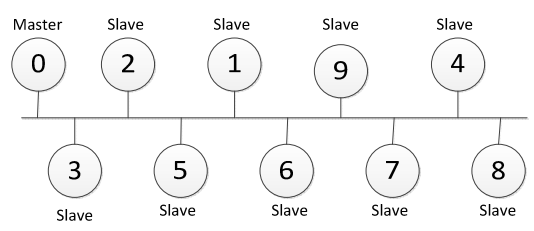

FIGURE I. NETWORK STRUCTURE.

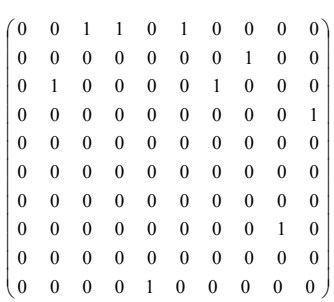

FIGURE II. TRANSMISSION MATRIX $\boldsymbol{M}$

For instance, as shown in Fig. 1, the master wants to send a message packet to the slave 8 . The slave 7 is the repeater that receives a message packet and retransmits it to the slave 8 , because the value of the element $M(7,8)$ of the transmission matrix, as shown in Fig. 2, is equal to one. The slave 1 is the repeater that receives a message packet and retransmits it to the slave 7 , since the value of the element $M(1,7)$ of the transmission matrix is equal to one. At last, the transmission path of the message packet from the mater to the slave 8 is determined: $0 \rightarrow 2 \rightarrow 1 \rightarrow 7 \rightarrow 8$.

\section{OPNET MODELLING}

OPNET Modeler simulator is a tool that can simulate the behaviour and performance of any type of network. As a high-end product in network programming, simulation and analysis tools, in the field of communication, national defence and data network, it has been widely accepted and used. It provides three layer modelling mechanisms. The bottom for the process model, constructs the algorithm agreement. The middle layer is the node model, reflecting the equipment of hardware features. The top is a network model, to show what the real network topology. According to the three simulation mechanism, we initialize the modelling and simulation of dynamic source routing algorithm, get the simulation data, and analysis of network performance.

\section{A. Communication Protocol Design}

Before three layer of model was carried out on the project, we need to design package modelling. Package is the basic unit of communication data, and is composed of a number of bytes. As shown in Fig. 4, we define each byte is eight bits, these bytes are respectively source address, relay address, destination address, control bits and data bits. The relay address block size is dynamic changing, according to the number of transmission relay address.

\section{B. Process Modelling}

Fig. 5 is the process of dynamic source routing algorithm simulation model diagram. The process simulation model is built by init, idle, snd, rcv, end, wt-free of six states. The function of the init state complete device initialization, including read the device name, master-slave state, device address, the beginning of the simulation time, statistic statements and generate the start of the interrupt command. Idle state is the system idle state in no event occurs. Snd state complete packets sent and statistic record package sent, after the program determine his own identity. when the package arrived, based on parameters of packet, such as carry information, their own status and address, rcv state accomplish packet forwarding, recording package receiving statistics, packet destruction, and renewal of the transfer matrix. wt free state is using CSMA protocol to monitor channel whether it is idle. During transmission of data, if the channel is idle, it will immediately send data. Else it will continue to monitor the channel, and don't send data until the channel is idle. end state is used to exact display initialization time at the end of the simulation. The two charts below are the flow chart of the algorithm of dynamic source routing, the flow chart of master as shown in Fig.6, the slaver process is shown in Fig.7.

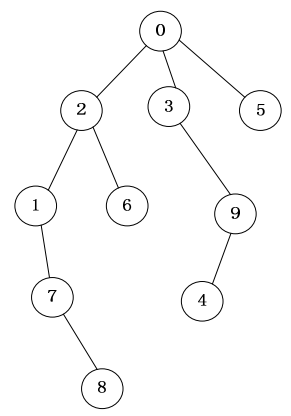

FIGURE III. NETWORK TOPOLOGY.

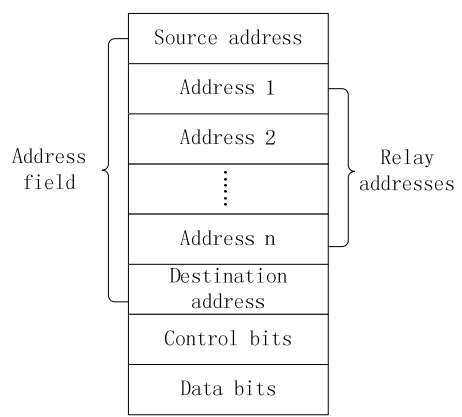

FIGURE IV. COMMUNICATION FRAME STRUCTURE.

MAX is number of the devices on the bus. M (MAX) is recorded broadcast array. When one element in the array is 1 , the master will send broadcast signals to the slaver whose address is equal to the corresponding element address, and waits to receive feedback response signal. When receiving the feedback, it will refresh the waiting time and continue to wait. N (MAX) is used to record the next layer to broadcast node equipment, when all of its data is 0 , the initialization process is over.

Slaver assign marks a value of 0 in the beginning. After the feedback response signal, mark assigned a value of 1 , stop feedback response signal. When it receives the broadcast signals whose destination address is equal to its 
own address, it will be broadcast, and it is the same as master broadcasting process. Here is not described in detail.

\section{Node Modelling}

Each device has the function of receiving and transmitting the packet. Therefore, each device equipped with a group of transceivers (bus_rx, bus_tx) and program processing module that is used to determine the receiving and forwarding (proc). Transceiver simulate hardware interface and control the transmission rate. The program module is used to load the program model, processing the received data. Dotted line is the statistical line which can listen to the state of the channel. As shown in Fig. 8.

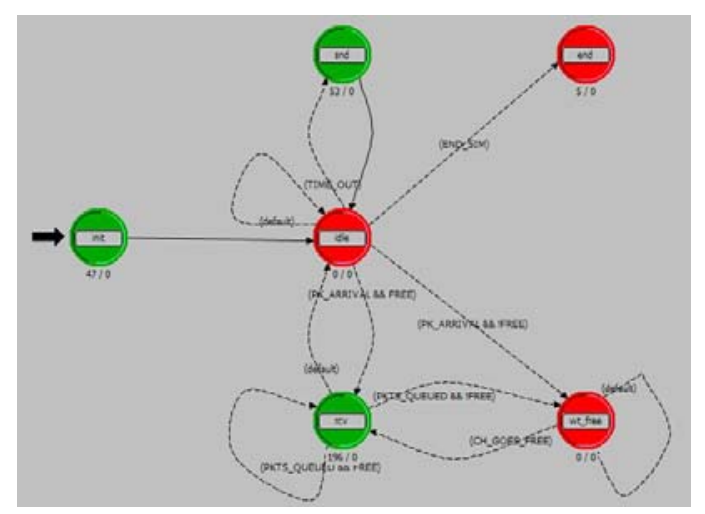

FIGURE V. PROCESS MODEL.

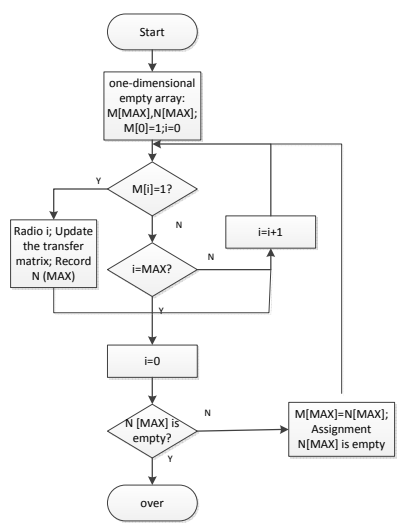

FIGURE VI. FLOW CHART OF THE MAST-CONTROLLER.

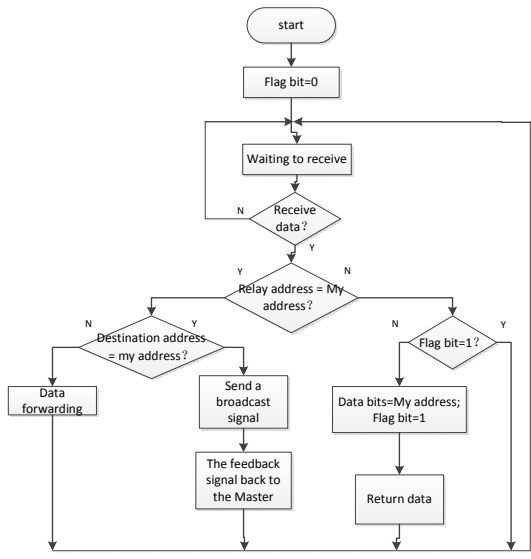

FIGURE VII. FLOW CHART OF THE SLAVE-CONTROLLER.

\section{The Network Structure Modelling}

As shown in Fig. 9, two kinds of equipment are the master and slave, they are connected to the bus. The master and slave devices is roughly the same, the only difference is the master can control bus, the slaver just response to the command. The unit length is $125 \mathrm{~m}$ in the picture below. The data transmission distance, set by program, is $250 \mathrm{~m}$, and it is equal to two units of length.

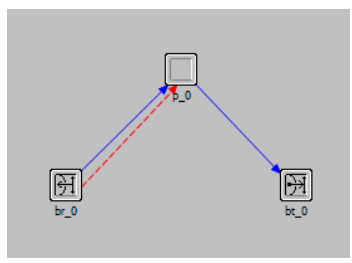

FIGURE VIII. NODE MODEL

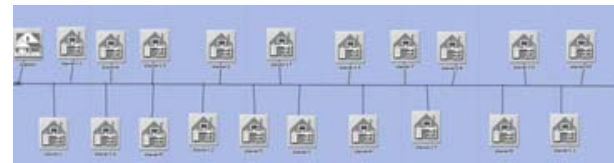

FIGURE IX. NETWORK MODEL.

\section{THE RESULTS OF SIMULATION ANALYSIS}

According to the 3.4 section a network model of the frame format and figure 3 , we made two experiments, respectively. The first set of experiments in order to ensure the quantity of 20 slavers unchanged, the data transmission speed is $600 \mathrm{bps}, 1200 \mathrm{bps}, 1800 \mathrm{bps}, 2400 \mathrm{bps}, 3600 \mathrm{bps}$, and $4800 \mathrm{bps}$. Simulation results are obtained corresponding to time $16.59 \mathrm{~s}, 8.29 \mathrm{~s}, 5.55 \mathrm{~s}, 4.18 \mathrm{~s}, 2.81 \mathrm{~s}$, and $2.09 \mathrm{~s}$.

The second set of experiments is to maintain the data transmission speed of 1200BPS under the same conditions, while the slaver number changed to $10,20,30,40,50$, respectively, corresponding simulation time results is $4.11 \mathrm{~s}$, $8.29 \mathrm{~s}, 13.08 \mathrm{~s}, 17.02 \mathrm{~s}, 21.03 \mathrm{~s}$.

According to the two groups of the experimental results, we turn them into the line chart which is shown in Fig. 10 and Fig.11. 


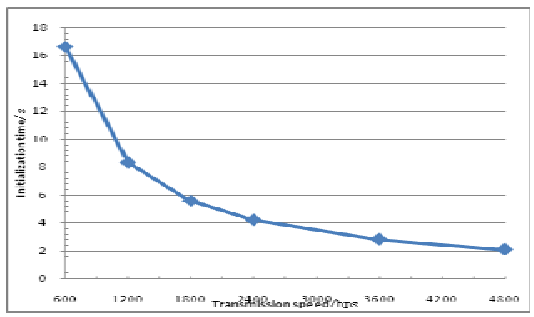

FIGURE X. TRANSMISSION

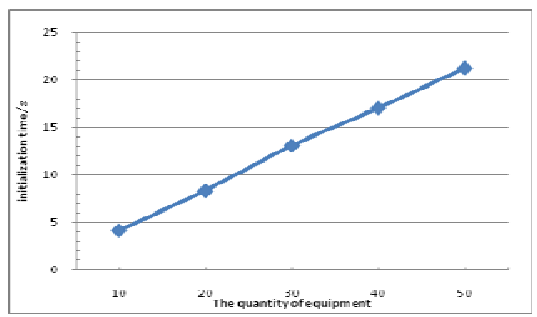

FIGURE XI. THE QUANTITY OF EQUIPMENT.

From the line as seen in Fig. 10, the relationship between transmission speed and data initialization time is the inverse function relation, the equipment for the number 20, their relation is about: initialization time $=9600 /$ transmission speed. From the line as seen in Fig. 11, the relationship between quantity of equipment and the system initialization time is a linear function relationship. The same as a 1200BPS in the transmission speed, their relation is about: initialization time $=0.42 *$ equipment number.

\section{CONCLUSION}

In view of the dynamic source routing algorithm, we use OPNET simulation approach, build a network model, drawing simulation results. Through the analysis of experimental results, we get the relationship between the data transmission speed and the system initialization time, and we also learn the influence for equipment quantity to initialization time, provides the corresponding experimental data for the actual network development and reference.

\section{REFERENCE}

[1] Qi Jia-jin, Chen Xue-ping, Liu Xiao-sheng, Advances of Research on Low-Voltage Power Line Carrier Communication Technology. Power System Technology, 34(5), pp. 161-171, 2010.

[2] Guo Junchang, Su Shiping, Fu Chuanzong, Research on Power Quality monitoring system based on low-voltage PLC technology. Proc. of the International Conference on Advanced Power System Automation and Protection, IEEE : Beijing, pp. 1574 - 1577, 2011.

[3] Wang Bo, Huang Pei-wei, Zhong You-ping, MAC Frame Design of Home Network Based On Low-Voltage PLC. Proc. of the International Conference on Industrial Technology, IEEE : Mumbai, pp. $2384-2389,2006$

[4] Liu Hai-tao, Zhang Bao-hui, Tan Lun-nong, Research on the channel capacity in a low voltage network channel. Journal of Xi'an Jiao tong University, 37(10), pp. 1048-1051, 2003.

[5] Liu Xiao-sheng, Zhang Liang, Zhou Yan, et al., Performance analysis of power line communication network model based on spider web. Proc. of the 8th International Conference on Power Electronics and ECCE Asia, IEEE : Jeju, pp. 953 - 959, 2011.

[6] Xue Wen-ling, Wang Zhen-chao, Gao Yue-hua, Research of dynamic routing based on improved Dynamic Ant Colony algorithm. Proc. of the 2nd International Conference on Industrial Mechatronics and Automation, IEEE : Wuhan, pp. 393 - 396, 2010.

[7] Zhang Liang, Liu Xiaosheng, Zhou Yan, et al., A Novel Routing Algorithm for Power Line Communication over a Low-voltage Distribution Network in a Smart Grid. Energies, 6(3), pp. 1421-1438, 2013.

[8] Wang Licheng, Gao Fei, Limited-cluster based networking algorithm of power line communication network for smart power grid. Proc. of the International Conference on Power System Technology, IEEE : Chengdu, pp. 2511-2516, 2014.

[9] Bauer Michael, Plappert Wolfgang, Wang Chong, Packet-oriented communication protocols for Smart Grid Services over low-speed PLC. Proc. of the International Symposium on Power Line Communications and Its Applications, IEEE : Dresden, pp. 89 - 94, 2009. 